\title{
PRELIMINARY RESULTS TOWARDS THE EQUIVALENCE OF TRANSFORMED CONTINUOUS-WAVE OPTICALLY STIMULATED LUMINESCENCE (CW-OSL) AND LINEARLY-MODULATED (LM-OSL) SIGNALS IN QUARTZ
}

\author{
GEORGE KITIS ${ }^{1}$, GEORGE S. POLYMERIS ${ }^{2}$, NAFIYE G. KIYAK ${ }^{2}$ and VASILIS PAGONIS ${ }^{3}$ \\ ${ }^{I}$ Nuclear Physics Laboratory, Aristotle University of Thessaloniki, 54124-Thessaloniki, Greece \\ ${ }^{2}$ IŞIK University, Faculty of Science and Arts, Physics Department, Şile 34980-Istanbul, Turkey \\ ${ }^{3}$ Physics Department, McDaniel College, Westminster, MD 21158, USA
}

Received 25 January 2010

Accepted 15 December 2010

\begin{abstract}
The present paper presents a comparative experimental study of two commonly measured Optically Stimulated Luminescence (OSL) signals in quartz. The experimental study measures both the continuous wave OSL (CW-OSL) and the linearly modulated (LM-OSL) signals from the same quartz sample for a range of stimulation temperatures between 180 and $280^{\circ} \mathrm{C}$, while the former is transformed to pseudo LM-OSL (ps LM-OSL). A computerized deconvolution curve analysis of the LM-OSL and ps LM-OSL signals was carried out, and the contributions of several OSL components to the initial OSL signal $(0.1 \mathrm{~s})$ were shown to be independent of the stimulation temperature used during the measurement. It was also found that the composite OSL $(0.1 \mathrm{~s})$ signal consists mainly of the first two OSL components present in the OSL curves. The equivalence of the ps LM-OSL (transformed CW-OSL) and of LM-OSL measurements was also examined by an appropriate choice of the experimental stimulation times, and of the stimulation power of the blue LEDs used during the measurement.
\end{abstract}

Keywords: OSL, transformed CW-OSL, LM-OSL, pseudo LM-OSL, quartz dating, OSL components, computerized OSL analysis.

\section{INTRODUCTION}

During applications of the optically stimulated luminescence (OSL) technique in dating and dosimetry, two methods of optical stimulation are commonly employed, namely continuous wave OSL (CW-OSL) and linearly modulated OSL (LM-OSL) (Bøtter-Jensen et al., 2003; Wintle and Murray, 2006; Bulur, 1996; Bulur et al., 2000).

While the pioneering OSL studies of Liritzis et al., (1997), Wintle and Murray (1999) and Murray and Win-

Corresponding author: G. S. Polymeris

e-mail: polymers@auth.gr tle (1999) were based mainly on measurements of the initial OSL (0.1 s) signal, several recent studies have attempted to identify and isolate the individual components that make-up the CW-OSL and LM-OSL signals from quartz (Bailey et al., 1997; Singarayer and Bailey, 2003; 2004; Jain et al., 2003; Kitis et al., 2007; Kiyak et al., 2007; 2008, Polymeris et al., 2008; 2009). Such studies are of major interest to the dating community, since it has been demonstrated that the medium and slow OSL components of quartz have the potential to be used for extending the range of OSL dating by one order of magnitude (Singarayer and Bailey, 2003).

It is desirable to use a well separated fast OSL component in luminescence dating protocols. Recently Hunt- 
ley (2006) pointed out that the separation of OSL components is independent of the stimulation mode used. Separation can take place by analytical as well as by instrumental procedures. In this framework, several procedures are reported (Kuhns et al., 2000; Chithambo and Galloway, 2001; Poolton et al., 2003; Jain and Lindvold, 2007; Wallinga et al., 2008). However, deconvolution of the pseudo LM-OSL decay curves is not reported in the relevant literature. Therefore, the main topic of this paper is the equivalence of LM-OSL and pseudo LM-OSL signals obtained by a transformation of the CW OSL signals, according to the procedure suggested by Bulur (1996). The theoretical equivalence between the peak shapes of LM-OSL and ps LM-OSL, simulated for general order kinetics, was shown by Kitis and Pagonis (2008). Later on, this study was also extended for the case of the physically meaningful mixed order OSL kinetics (Kitis et al., 2009). Having established the aforementioned theoretical equivalence, the experimental aspects of the equivalence are studied within the framework of a complicated experimental protocol involving not only severe but also repeated external treatments of the quartz samples, which can alter substantially the shapes of both LM-OSL and CW-OSL curves. For this reason a quite complicated protocol (almost identical to that used in the work of Murray and Wintle, 1999) was chosen in order to verify the equivalence as a function of external experimental parameters.

In this paper a comparative study of ps LM-OSL (transformed CW-OSL) and LM-OSL signals in quartz is presented. The specific goals of the present work are the following:

1) Examine the relation of the initial OSL ( $0.1 \mathrm{~s})$ signal with the various OSL components of quartz, by performing a computerized deconvolution curve analysis (CDCA) of the ps LM-OSL and LM-OSL signals. This examination is carried out for a large range of isothermal stimulation temperatures and isothermal times.

2) Demonstrate the complete equivalence of LM-OSL and CW-OSL data by appropriate choices of the stimulation times and stimulation intensities.

\section{SAMPLES AND EXPERIMENTAL PROCEDURE}

All measurements were performed using the automated Risø TL/OSL reader (model TL/OSL-DA-15), which has an internal ${ }^{90} \mathrm{Sr} /{ }^{90} \mathrm{Y}$ beta ray source of dose rate $\sim 0.1 \mathrm{~Gy} / \mathrm{s}$. Blue light emitting diodes (LEDs) $(470 \mathrm{~nm}$, $40 \mathrm{~mW} / \mathrm{cm}^{2}$ ) were used for stimulation and the OSL signal was detected through a $7.5 \mathrm{~mm}$ thick Hoya U-340 filter. The sample studied was one of sedimentary origin (laboratory reference PDK) collected from the coastal area of the Sea of Marmara, in the Asian part of Turkey (Kiyak and Canel, 2006).

The basic multiple aliquot experimental protocol used in the present work is as follows:
Step 1: Bleach stage: Blue light stimulation at $125^{\circ} \mathrm{C}$ for $100 \mathrm{~s}$

Step 2: Give laboratory dose of $51 \mathrm{~Gy}$ at $20^{\circ} \mathrm{C}$

Step 3: Heat the sample to a temperature $T_{i}=180^{\circ} \mathrm{C}$ at a heating rate of $1^{\circ} \mathrm{C} / \mathrm{s}$ and keep sample at temperature $T_{i}$ for time $t_{j}=10 \mathrm{~s}$

Step 4: Give small test dose of 0.2 Gy at $20^{\circ} \mathrm{C}$

Step 5: Heat to $160^{\circ} \mathrm{C}$ and measure sensitivity using the $110^{\circ} \mathrm{C}$ TL peak in quartz after heating at $1^{\circ} \mathrm{C} / \mathrm{s}$. Record TL $\left(110^{\circ} \mathrm{C}\right)$ signal

Step 6: Continuous wave (CW) blue light stimulation for $355 \mathrm{~s}$ at the stimulation temperature of $125^{\circ} \mathrm{C}$. Record CW-OSL (355 s) signal

Step 7: Repeat steps 2-6 using the same aliquot for all isothermal times $t_{i}=10,20,50,100,250,500,1000,2000$, $5000 \mathrm{~s}$, where $t_{i}$ denotes the varying duration of isothermal TL before OSL measurement.

Step 8: Repeat steps 2-7 using a different aliquot for each preheat temperature $T_{i}=180,200,220,240,260,280^{\circ} \mathrm{C}$. A total of 6 aliquots were used in this study.

In a second experiment the same protocol was used to measure the LM-OSL signal by replacing Step 6 in the above protocol with the following:

Step 6a: Linearly modulated (LM) blue stimulation for $500 \mathrm{~s}$ at a stimulation temperature of $125^{\circ} \mathrm{C}$. Record LM-OSL (500 s) signal

It is to be noted that the $110^{\circ} \mathrm{C}$ TL intensity recorded during step 5 is used for normalization. Finally, the different CW-OSL and LM-OSL time intervals (355 s and $500 \mathrm{~s}$ in steps 6 and $6 \mathrm{a}$ above) were chosen intentionally so that a direct comparison of the CW-OSL and LM-OSL measurements can be carried out at the different temperatures. These choices are explained in detail in Appendix A.

\section{METHODS OF ANALYSIS}

\section{Analysis of the OSL (0.1 s) signal}

Several studies have shown that the initial $0.1 \mathrm{~s}$ of an OSL signal in quartz is correlated with the fast OSL component (Bøtter-Jensen et al., 2003). The goal of this study is to investigate the composition of the OSL $(0.1 \mathrm{~s})$ signal and to find what percentage of the OSL $(0.1 \mathrm{~s})$ signal comes from each of the OSL components that contribute to it. The methodology used was as follows. The CW-OSL and LM-OSL curves obtained at Steps 6 and $6 \mathrm{a}$ of the protocol were deconvoluted into individual components. Once the individual LM-OSL components were obtained, the computerized analysis is used to find the realtive contribution of each LM-OSL component to the initial OSL ( $0.1 \mathrm{~s})$ signal.

It is noted that in the case of LM-OSL measurements, all OSL components $C_{i}$ start from $t \approx 0$. This means that the OSL $(0.1 \mathrm{~s})$ signal is the sum of all LM-OSL components at $t=0$ i.e. 


$$
\operatorname{OSL}(0.1 s)=\sum_{i} C_{i}(t \approx 0)
$$

\section{Analysis of the LM-OSL data}

The stimulation intensity during an LM-OSL experiment is given by

$$
I(t)=I_{L M} \cdot \frac{t}{P_{L M}} \quad\left(t=0 \ldots P_{L M}\right),
$$

where $P_{L M}$ denotes the duration of the LM-OSL measurement and $I_{L M}$ is the light intensity reached at the end of the measurement. Under these conditions, the total energy $E_{L M}$ delivered to the sample using LM-OSL is given by the simple integration:

$$
E_{L M}=\int_{0}^{P_{L M}} I(t) d t=\int_{0}^{P_{L M}} I_{L M} \frac{t}{P_{L M}} d t=\frac{1}{2} I_{L M} P_{L M}
$$

The LM-OSL curves were deconvoluted using a first order kinetics expression proposed by Bulur (1996). This expression was further transformed recently by Kitis and Pagonis (2008) into another expression containing only the peak maximum intensity $I_{m}$ and the corresponding time $t_{m}$. These two variables can be extracted directly from the experimental OSL curves. The modified expression used in our computerized procedure is:

$$
I(t)=1.6487 \cdot I_{m} \cdot \frac{t}{t_{m}} \cdot \exp \left[-\frac{t^{2}}{2 t_{m}^{2}}\right]
$$

The background signal was simulated by an equation of the form

$$
G_{L M}(t)=A+c \cdot t,
$$

where $A$ is the average in the first few seconds of a zero dose LM-OSL measurement resulting from both the stimulation light and the dark counts off the detector, while $c$ is a constant.

\section{Analysis of the CW-OSL data}

The stimulation intensity $I_{s t}$ during a CW-OSL experiment is constant and given by

$$
I_{S T}(t)=I_{C W} \quad\left(t=0 \ldots P_{C W}\right)
$$

where $P_{C W}$ denotes the duration of the CW-OSL measurement and $I_{C W}$ is the constant light intensity. Since the light intensity is constant, the total energy $E_{C W}$ delivered to the sample using CW-OSL is given by the product:

$$
E_{C W}=I_{C W} P_{C W}
$$

For the deconvolution analysis of the CW-OSL curves, these were transformed into peak-shaped pseudo-
LM-OSL (ps-LM-OSL) curves using the transformations introduced by Bulur (1996). In these transformations a new time-dependent variable is defined by the expression

$u=\sqrt{2 \cdot t \cdot P_{C W}}$,

where $P_{C W}$ is the total duration of the CW-OSL stimulation. Using this transformation the featureless CW-OSL decay $I(t)$ is transformed into the following peak-shaped ps-LM-OSL intensity $I(u)$ :

$$
I(u)=u \cdot \frac{I(t)}{P_{C W}}
$$

The total time $P_{P S-L M}$ for the transformed ps-LM-OSL curve is obtained from Eq. 3.6 by setting $t=P_{C W}$ to obtain

$$
P_{P S-L M}=\sqrt{2 t P_{C W}}=\sqrt{2} P_{C W}
$$

For deconvolution purposes the single peak expression Eq. 3.7 of $I(u)$ is identical to the expression in Eq. 3.3 where the $t$ and $t_{m}$ variables are replaced by $u$ and $u_{m}$ i.e. (Polymeris et al., 2006):

$$
I(u)=1.6487 \cdot I_{m} \cdot \frac{u}{u_{m}} \cdot \exp \left[-\frac{u^{2}}{2 u_{m}^{2}}\right]
$$

The background signal in the case of ps-LM-OSL was simulated by an equation of the form

$$
G_{P S}(t)=A_{0}+B \cdot \frac{t}{P},
$$

Where $A_{0}$ accounts for the additional background from the dark counts, and $B$ is the average of a zero-dose CW-OSL measurement.

It is noted that the values of $A, B$ and $c$ in the background functions are not left to vary arbitrarily during the deconvolution process. Instead, zero dose LM-OSL and CW-OSL curves were experimentally obtained and fitted with the background Eqs. 3.4 and 3.10. During the deconvolution procedure these quantities were left to vary within their evaluated experimental errors. All curve fittings were performed using the MINUIT computer program (James and Roos, 1977), while the goodness of fit was tested using the Figure Of Merit (FOM) of Balian and Eddy (1977) given by:

$$
F O M=\sum_{i} \frac{\left|Y_{\text {Exper }}-Y_{F i t}\right|}{A},
$$

where $Y_{\text {Exper }}$ is a point on the experimental glow-curve, $Y_{F i t}$ is a point on the fitted glow-curve and $A$ is the area of the fitted curve. The FOM values obtained were between $0.8 \%$ and $4 \%$ depending upon the statistics. 


\section{EXPERIMENTAL RESULTS}

\section{Deconvolution of the experimental OSL curves}

Examples of deconvoluted experimental OSL curves are shown in Figs. 1 and 2. Fig. 1 indicates a deconvolution example of an LM-OSL curve with a total simulation time of $P_{L M}=500 \mathrm{~s}$, which was fitted using four first order kinetic components. Only components 1,2 and 3 are clearly resolved in the experimental data. These components will be referred to as $\mathrm{C}_{1}, \mathrm{C}_{2}, \mathrm{C}_{3}$ in the rest of this paper. Component 4 represents the sum of all other single components beyond component 3 .

The inset to Fig. 2 shows an example of the original experimental CW-OSL data which was measured over a time interval of $P_{C W}=355 \mathrm{~s}$, as previously discussed. This $\mathrm{CW}-\mathrm{OSL}$ curve was transformed into a ps-LM-OSL

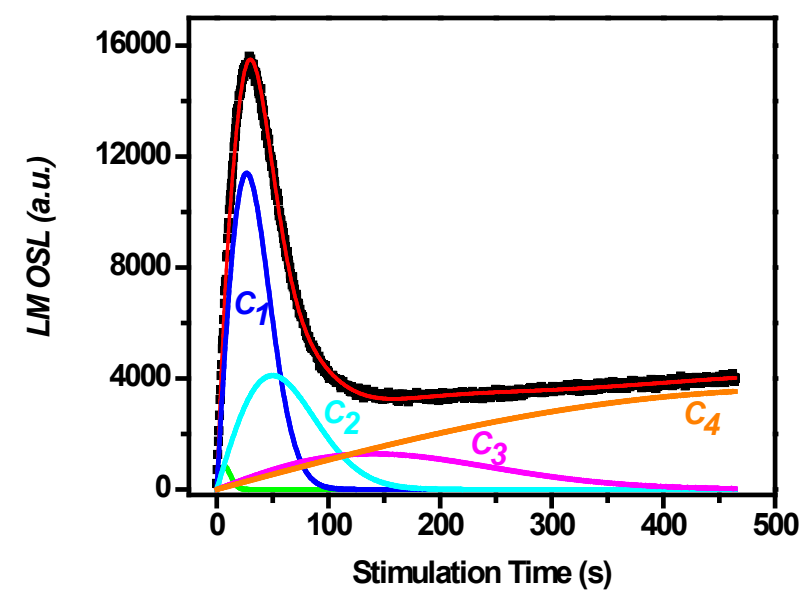

Fig. 1. CDCA of an LM-OSL curve showing the individual OSL components.

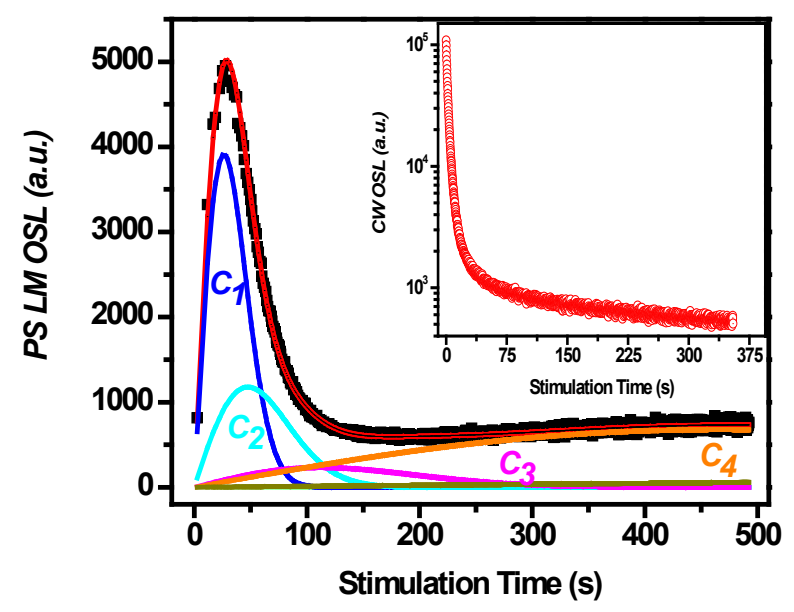

Fig. 2. $C D C A$ of a $P S-L M-O S L$ curve resulting from the transformation of the respective CW-OSL data (inset). peak-shaped graph and its component analysis is shown in main frame of Fig. 2. Comparison of the results in Figs. 1 and 2 shows that the analysis of the ps LM-OSL data yields exactly the same results as the analysis of the LM-OSL data when the experimental conditions are chosen properly. This is discussed further in the next section.

We first investigate the relationship between the OSL $(0.1 \mathrm{~s})$ signal and the individual OSL components. The results are shown in the set of Figs. 3-5 where the upper panel of each figure corresponds to the LM-OSL results while the lower panel corresponds to the ps LM-OSL results. In all cases the behaviour of the OSL $(0.1 \mathrm{~s})$ signal is in good agreement with component $\mathrm{C}_{1}$ of the LMOSL signal, and is almost identical to that of component $\mathrm{C}_{1}$ of the ps LM-OSL signal. The agreement between the OSL $(0.1 \mathrm{~s})$ signal and component $\mathrm{C}_{2}$ of both LM-OSL and ps LM-OSL is very good only at a stimulation temperature of $180^{\circ} \mathrm{C}$, and becomes poor as the stimulation temperature increases. On the other hand, there is no discernible correlation between OSL component $\mathrm{C}_{3}$ and the OSL $(0.1 \mathrm{~s})$ signal at any stimulation temperature.

The ratio of the OSL $(0.1 \mathrm{~s})$ signal over the integral of component $\mathrm{C}_{1}$ of both LM-OSL and ps-LM-OSL is shown in Table 1, together with the corresponding ratio over the OSL component $\mathrm{C}_{2}$. As it is seen from Table 1, these ratios show an excellent stability for all measurements (LM-OSL or CW-OSL) and at all stimulation temperatures, indicating that the OSL $(0.1 \mathrm{~s})$ signal corresponds to $\sim 7.5 \%$ of the total fast OSL component $\mathrm{C}_{1}$, and $\sim 11.5 \%$ for component $\mathrm{C}_{2}$.

The second part of the present investigation is to find how much each component $\mathrm{C}_{1}, \mathrm{C}_{2}$ and $\mathrm{C}_{3}$ contributes to the OSL $(0.1 \mathrm{~s})$ signal. From a theoretical point of view, all the OSL components start from $t \approx 0$. Therefore, the ratio of each component at $t \approx 0$ over the sum of Eq. 3.1 will give the contribution of each component to the OSL (0.1 s) signal.

Table 2 shows that the OSL $(0.1 \mathrm{~s})$ signal comes mainly from components $C_{1}$ and $C_{2}$, with a very small contribution around $2 \%$ coming from the slower components $\mathrm{C}_{3}$ and $\mathrm{C}_{4}$. The main contribution of about $85 \%$ comes from the component $\mathrm{C}_{1}$ and a secondary contribution of about $13 \%$ comes from the component $\mathrm{C}_{2}$. The results are the same for both LM-OSL and CW-OSL and for all stimulation temperatures.

Table 1. Percentage ratios of the OSL $(0.1 \mathrm{~s})$ signal over the integral of the components $C_{1}$ and $C_{2}$ of both $L M$ and ps-LM-OSL data.

\begin{tabular}{lcccc}
\hline $\left.\mathbf{T}^{\circ} \mathbf{C}\right)$ & LM-C $_{1}$ & PSLM-C $_{1}$ & LM-C $_{2}$ & PSLM-C $_{2}$ \\
\hline 180 & $7.70 \pm 0.26$ & $6.60 \pm 0.14$ & $11.46 \pm 0.40$ & $12.57 \pm 0.70$ \\
200 & $7.70 \pm 0.14$ & $6.80 \pm 0.30$ & $11.14 \pm 0.22$ & $11.98 \pm 0.80$ \\
220 & $7.30 \pm 0.25$ & $7.50 \pm 0.20$ & $11.43 \pm 0.60$ & $11.45 \pm 0.80$ \\
240 & $7.70 \pm 0.14$ & $6.50 \pm 0.50$ & $10.82 \pm 0.24$ & $12.30 \pm 1.20$ \\
260 & $7.30 \pm 0.50$ & $6.40 \pm 0.30$ & $13.2 \pm 1.60$ & $11.10 \pm 1.80$ \\
280 & $7.00 \pm 0.20$ & $5.80 \pm 0.05$ & $10.2 \pm 1.00$ & $11.30 \pm 0.70$ \\
\hline
\end{tabular}




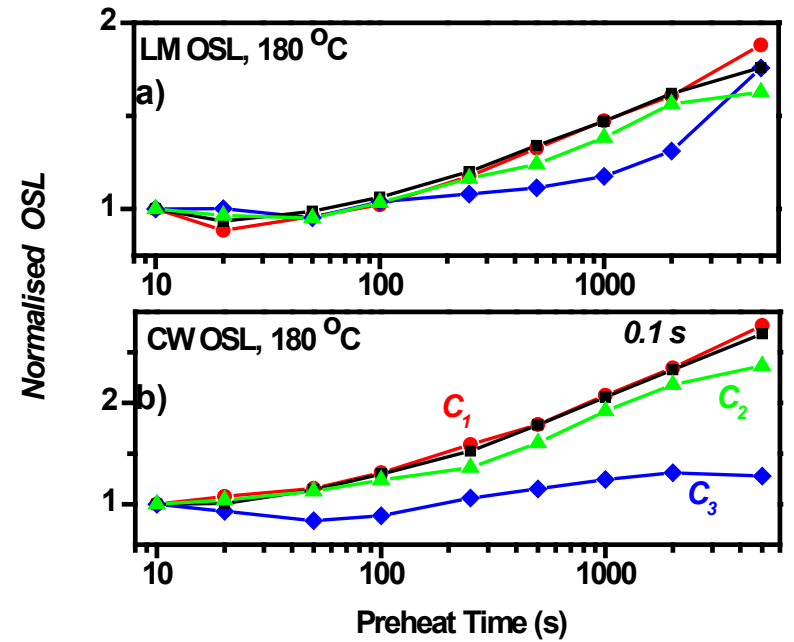

Fig. 3. Normalized response of the $0.1 \mathrm{~s}$ OSL signal and of the components 1, 2 and 3 of the (a) LM-OSL and (b) of the ps LM-OSL curves at a stimulation temperature of $\mathrm{T}=180^{\circ} \mathrm{C}$.

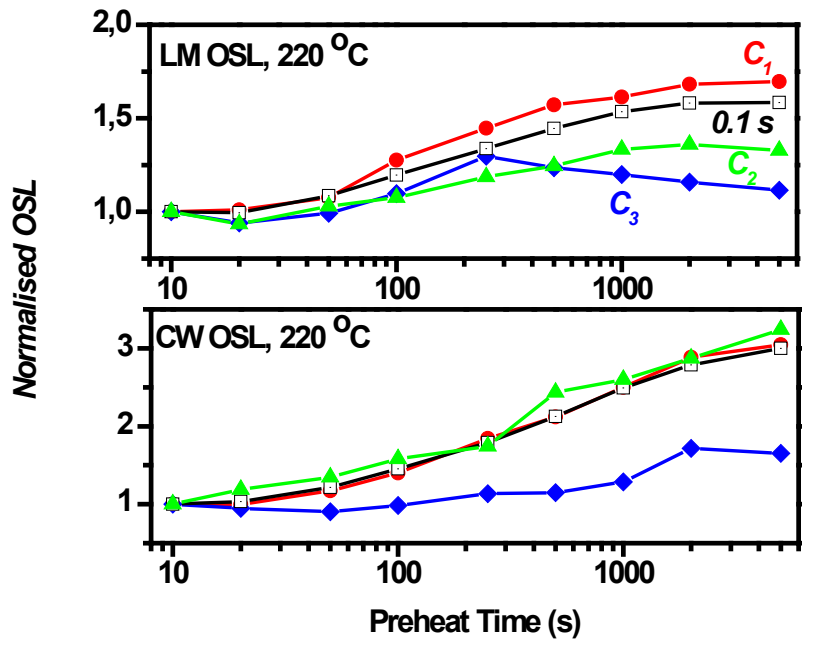

Fig. 4. Same as in Fig. 3 with the LM-OSL and CW-OSL measurements carried out at a stimulation temperature of $220^{\circ} \mathrm{C}$.

These results are identical to the results presented in Tables 1 and 2 of Murray and Wintle (1999), who found 3 main components for their OSL ( $0.1 \mathrm{~s})$ signal, labelled $\mathrm{A}, \mathrm{B}$ and $\mathrm{C}$. The OSL signal for their untreated natural quartz sample contained contributions of $99 \%$ and $1 \%$ of components $\mathrm{A}$ and $\mathrm{C}$ correspondingly. The OSL signal of their bleached-irradiated samples contained contributions of $61 \%, 38 \%$ and $1 \%$ from components $\mathrm{A}, \mathrm{B}, \mathrm{C}$ respectively.

\section{Equivalence of the CW-OSL and LM-OSL signal analysis}

In this section we show that the experimental shapes of ps LM-OSL and LM-OSL curves are essentially iden-

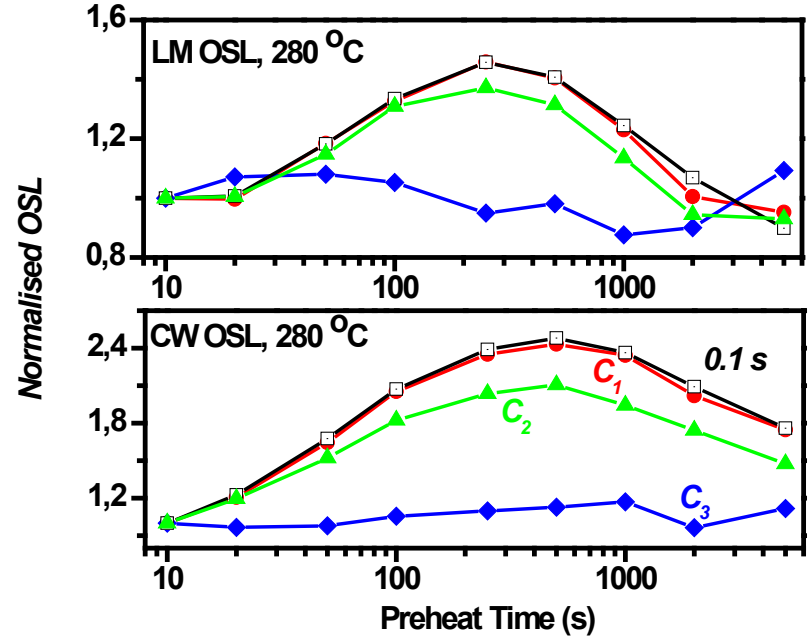

Fig. 5. Same as in Fig. 4 with the LM-OSL and CW-OSL measurements carried out at a stimulation temperature of $\mathrm{T}=280^{\circ} \mathrm{C}$.

Table 2. Percentage ratios of the components $C_{1}$ and $C_{2}$ at $t \approx 0$ over the sum of all components $C_{1}-C_{4}$

\begin{tabular}{|c|c|c|c|c|}
\hline $\mathrm{T}\left({ }^{\circ} \mathrm{C}\right)$ & LM-C 1 & PSLM-C $_{1}$ & LM-C ${ }_{2}$ & PSLM-C \\
\hline$\frac{1}{180}$ & $84.3 \pm 1.6$ & $81.9 \pm 1.5$ & $13.5 \pm 1.5$ & $14.8 \pm 1.1$ \\
\hline 200 & $84.8 \pm 0.6$ & $84.7 \pm 0.8$ & $13.5 \pm 0.6$ & $13.4 \pm 0.7$ \\
\hline 220 & $85.9 \pm 1.0$ & $85.9 \pm 1.0$ & $12.7 \pm 1.1$ & $11.3 \pm 0.5$ \\
\hline 240 & $86.9 \pm 0.9$ & $84.6 \pm 1.0$ & $11.7 \pm 1.1$ & $13.8 \pm 1.3$ \\
\hline 260 & $87.3 \pm 1.4$ & $87.3 \pm 2.2$ & $11.7 \pm 1.5$ & $11.4 \pm 2.4$ \\
\hline 280 & $84.7 \pm 0.3$ & $83.1 \pm 1.3$ & $14.5 \pm 0.4$ & $15.3 \pm 1.1$ \\
\hline
\end{tabular}

tical and that they also yield the same type of information under the chosen experimental conditions.

The left-hand panel of Fig. 6 shows typical results showing good agreement between the experimental LMOSL and the corresponding experimental ps LM-OSL curves measured on the same sample. This was the case of stimulation at $180^{\circ} \mathrm{C}$. The middle panel of the same Fig. 6 presents the respective results for stimulation at $220^{\circ} \mathrm{C}$, while the right hand panel shows one of the worst cases, after stimulating at $280^{\circ} \mathrm{C}$, where the two sets disagree with each other, especially as the stimulation time increases. The slight difference at higher stimulation times is probably attributed to the different background signals in each measurement.

Our analysis of the data showed that deconvolution analysis of both LM-OSL and their counterpart ps LMOSL curves give exactly the same results. According to our theoretical choice of the experimental settings discussed in Appendix A, the individual peaks resulting from the deconvolution must have the same peak maximum time (i.e. $\mathrm{t}_{\mathrm{m}}=\mathrm{u}_{\mathrm{m}}$ ). Table 3 shows the values of the peak maxima $t_{m}$ and $u_{m}$ for components $C_{1}, C_{2}$ and $C_{3}$ as they are obtained from a separate analysis. There is good agreement between the maxima obtained from analysing the two independently measured sets of data. The last 

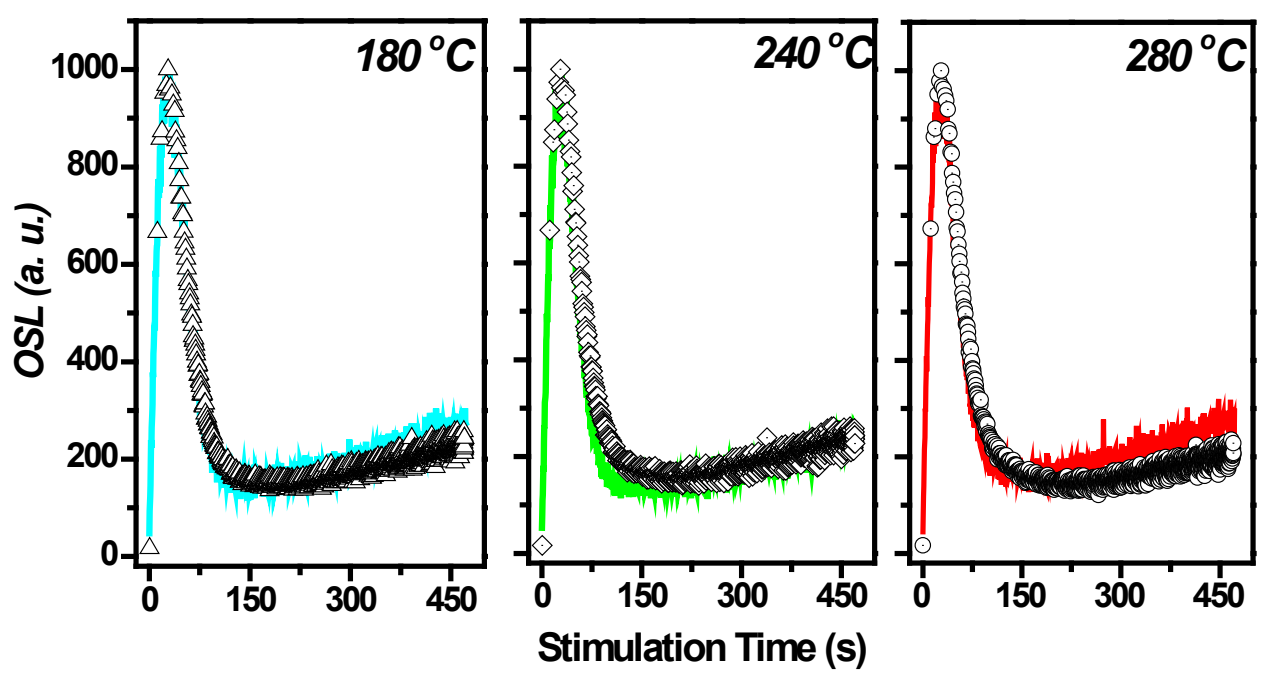

Fig. 6. LM-OSL and $p s-L M-O S L$

curves after stimulation at $180^{\circ} \mathrm{C}$ (left hand side panel), at $220^{\circ} \mathrm{C}$ (middle panel) as well as at $280^{\circ} \mathrm{C}$ (right hand side panel), normalized to the sample peak height. Open points correspond to the LM-OSL and continuous lines to the $p s-L M$ OSL curves.

column in Table 3 shows the ratio of the area under individual LM-OSL peaks over the corresponding ps LMOSL peak integrals; these ratios are indeed the same for all analysed curves. It is noted that the ratios on the last column of Table 3 are not unity, because the ps LM-OSL curve represents a transformation of the original data according to Eq. 3.6. Ratios of LM-OSL and ps-LM-OSL integrated intensities are not expected to be of the order of unity. On the contrary, ratios of the LM-OSL to the corresponding CW-OSL (after re-transformation of the ps LM-OSL curve to the corresponding CW-OSL) are expected to be close to unity.

In the case of component $\mathrm{C}_{3}$ there is an appreciable variation in Table 3 due to the experimental uncertainties at the high stimulation times of the OSL curves. The high stimulation time part of both LM and CW-OSL curves is difficult to reproduce accurately, since it is the sum of the tails of all OSL components beyond component $\mathrm{C}_{3}$, whose behaviour is unknown.

Our results are in agreement with the recent theoretical and experimental study by Wallinga et al. (2008), who found that the OSL signal in several samples was not affected by the stimulation mode, and that there is a close correspondence between $\mathrm{CW}, \mathrm{LM}$ and hyperbolically modulated OSL data.

Table 3. Results of independent computerized deconvolution analysis of the LM-OSL and ps-LM-OSL data.

\begin{tabular}{lccc}
\hline Comp. & $\mathrm{t}_{\mathrm{m}}(\mathbf{s})$ & $\mathrm{u}_{\mathrm{m}}(\mathbf{s})$ & (LM-area)/(ps-LM-area) \\
\hline $\mathrm{C}_{1}$ & $27.01 \pm 0.60$ & $27.3 \pm 1.1$ & $3.42 \pm 0.30$ \\
$\mathrm{C}_{2}$ & $50.9 \pm 2.3$ & $51.9 \pm 4.2$ & $2.25 \pm 0.20$ \\
$\mathrm{C}_{3}$ & $145.0 \pm 7.1$ & $148 \pm 10$ & $1.89 \pm 0.80$ \\
\hline
\end{tabular}

\section{CONCLUSIONS}

The OSL $(0.1 \mathrm{~s})$ signal is found to be a composite signal consisting $\sim 85 \%$ of OSL component $\mathrm{C}_{1}$ centred at $t_{m} \sim 27 \mathrm{~s}$ and with a smaller contribution of $\sim 13 \%$ from OSL component $\mathrm{C}_{2}$ centred at $t_{m} \sim 51$ s. Computerized analysis of all LM-OSL and ps LM-OSL curves at stimulation temperatures between 180 and $280^{\circ} \mathrm{C}$ showed that these percentages are independent from the stimulation temperature.

Furthermore, it is possible to transform CW-OSL data into the corresponding ps LM-OSL data measured on the same sample by choosing appropriately the experimental values of the stimulation intensities and total stimulation times. Computerized analysis of all LM-OSL and ps LMOSL curves showed that these two modes of OSL stimulation yield exactly the same information, showing the equivalence between LM-OSL and CW-OSL measurements. Further work is required in order to apply the same study to numerous quartz samples with various LM as well as CW-OSL curve shapes.

\section{ACKNOWLEDGEMENT}

George S. Polymeris is financially supported by TUBITAK (The Scientific and Technological Research Council of Turkey), in the framework of a Post-doc Fellowship for foreign citizens.

\section{REFERENCES}

Bailey RM, Smith BW, Rhodes EJ, 1997. Partial bleaching and the decay form characteristics of quartz OSL. Radiation Measurements 27(2): 123-136, DOI 10.1016/S1350-4487(96)00157-6.

Balian HM, Eddy NW, 1977. Figure-of-merit (FOM): An improved criterion over the normalized Chi-squared test for assessing goodness-of-fit of gamma-ray spectral peaks. Nuclear Instruments and Methods 145(2): 389-395, DOI 10.1016/0029-554X(77)90437-2.

Bøtter-Jensen L, McKeever SWS and Wintle AG, 2003. Optically Stimulated Luminescence Dosimetry. Elsevier, Amsterdam: 336 pp 
Bulur E, 1996. An alternative technique for optically stimulated luminescence (OSL) experiment. Radiation Measurements 26(5): 701709, DOI 10.1016/S1350-4487(97)82884-3.

Bulur E, Bøtter-Jensen L and Murray AS, 2000. Optically stimulated luminescence from quartz measured using the linear modulation technique. Radiation Measurements 32(5-6): 407-411, DOI 10.1016/S1350-4487(00)00115-3.

Chithambo M L and Galloway R B, 2001. On the slow component of luminescence stimulated from quartz by pulsed blue light-emitting diodes. Nuclear Instruments and Methods in Physics Research, Section B 183(3-4): 358-368, DOI 10.1016/S0168583X(01)00694-2.

Jain $\mathrm{M}$ and Lindvold LR, 2007. Blue light stimulation and linearly modulated optically stimulated luminescence. Ancient TL 25: 6980

Jain M, Murray AS and Bøtter-Jensen L, 2003. Characterisation of blue light stimulated luminescence components in different quartz samples: implications for dose measurement. Radiation Measurements 37(4-5): 441-449, DOI 10.1016/S1350-4487(03)00052-0.

James $\mathrm{F}$ and Roos M, 1977. MINUIT, CERN program library entry D506 http://consult.cern.ch/writeups/minuit.

Huntley DJ, 2006. Thoughts arising from "Choi, Duller and Wintle: analysis of quartz LM-OSL curves. Ancient TL 24, 9 - 20 (2006)." Ancient TL 24: 69-70.

Kitis G, Furetta C and Pagonis V, 2009. Mixed order kinetics model for optically stimulated luminescence. Modern Physics Letters B 23(27): 3191-3207,.DOI 10.1142/S0217984909021351.

Kitis G and Pagonis V, 2008. Computerized curve deconvolution analysis for LM-OSL. Radiation Measurements 43(2-6): 737-741, DOI 10.1016/j.radmeas.2007.12.055.

Kitis G, Polymeris GS and Kiyak NG, 2007. Component resolved thermal stability and recuperation study of the LM-OSL curves of four sedimentary quartz samples. Radiation Measurements 42(8): 1273-1279, DOI 10.1016/j.radmeas.2007.05.050.

Kiyak NG and Canel T, 2006. Equivalent dose in quartz from young samples using the SAR protocol and the effect of preheat temperature. Radiation Measurements 41(7-8): 917-922, DOI 10.1016/j.radmeas.2006.04.006.

Kiyak NG, Polymeris GS and Kitis G, 2007. Component resolved OSL dose response and sensitization of various sedimentary quartz samples. Radiation Measurements 42(2): 144-155,.DOI 10.1016/j.radmeas.2007.02.052

Kiyak NG, Polymeris GS and Kitis G, 2008. LM-OSL thermal activation curves of quartz: relevance to the thermal activation of the $110^{\circ} \mathrm{C}$ TL glow-peak. Radiation Measurements 43(2-6): 263-268, DOI 10.1016/j.radmeas.2007.12.039.
Kuhns CK, Agersnap Larsen N, McKeever, SWS, 2000. Characteristics of LM-OSL from several different types of quartz. Radiation Measurements 32(5-6): 413-418, DOI 10.1016/S13504487(00)00065-2.

Liritzis I, Galoway RB and Hong DG, 1997. Single aliquot of ceramics by green light stimulation of luminescence from quartz. Nuclear Instruments and Methods in Physics Research B 132(3): 457-467, DOI 10.1016/S0168-583X(97)00456-4.

Murray AS and Wintle AG, 1999. Isothermal decay of optically stimulated luminescence in quartz . Radiation Measurements 30(1): 119-125, DOI 10.1016/S1350-4487(98)00097-3.

Polymeris GS, Tsirliganis, NC, Loukou Z and Kitis G, 2006. A comparative study of anomalous fading effects of TL and OSL signals of Durango apatite. Physica Status Solidi A 203(3): 578-590, DOI 10.1002/pssa.200521347.

Polymeris GS, Kiyak NG and Kitis G, 2008. Component resolved bleaching study of the blue LM-OSL signal of various quartz samples. Geochronometria 32: 79-85, DOI 10.2478/v10003-008-00283.

Polymeris GS, Afouxenidis D, Tsirliganis NC and Kitis G, 2009. The $\mathrm{TL}$ and room temperature OSL properties of the glow peak at $110^{\circ} \mathrm{C}$ in natural milky quartz: A case study. Radiation Measurements 44(1): 23-31, DOI 10.1016/j.radmeas.2008.10.007.

Poolton NRJ, Bøtter-Jensen, L., Andersen CE, Jain M, Murray AS, Malins AER and Quinn FM, 2003. Measuring modulated luminescence using non-modulated stimulation: ramping the sampling period. Radiation Measurements 37(6): 639-645, DOI 10.1016/S1350-4487(03)00244-0.

Singarayer JS and Bailey RM, 2003. Further investigations of the quartz optically stimulated luminescence components using linear modulation. Radiation Measurements 37(4-5): 451-458, DOI 10.1016/S1350-4487(03)00062-3.

Singarayer JS and Bailey RM, 2004. Component-resolved bleaching spectra of quartz optically stimulated luminescence: preliminary results and implications for dating. Radiation Measurements 38(1): 111-118, DOI 10.1016/S1350-4487(03)00250-6.

Wallinga J, Bos AJJ and Duller GAT, 2008. On the separation of quartz OSL signal components using different stimulation modes. Radiation Measurements 43(2-6): 742-747, DOI 10.1016/j.radmeas.2008.01.013.

Wintle AG and Murray AS, 1999. Luminescence sensitivity changes in quartz. Radiation Measurements 30(1): 107-118, DOI 10.1016/S1350-4487(98)00096-1.

Wintle AG and Murray AS, 2006. A review of quartz optically stimulated luminescence characteristics and their relevance in singlealiquot regeneration dating protocols. Radiation Measurements 41(4): 369-391, DOI 10.1016/j.radmeas.2005.11.001. 


\section{APPENDIX A}

\section{Choice of experimental conditions for LM-OSL and CW-OSL measurements}

One might expect that analysis of a ps-LM-OSL curve would give the same results as an analysis of the corresponding LM-OSL curve measured on the same sample. In this section we show that the stimulation intensity and the total simulation time during the LM-OSL and CWOSL experiments can be chosen so that the ps-LM-OSL and LM-OSL curves are identical.

As a first requirement for the two peak-shaped curves to be identical, the total time for the LM-OSL measurement $P_{L M}$ is required to be equal to the corresponding total time $P_{P S-L M}$ for the PS-LM-OSL transformed data, i.e. $P_{P S-L M}=P_{L M}$. By using Eq. 3.8 this yields

$$
P_{P S-L M}=P_{L M}=\sqrt{2} P_{C W}
$$

This is our first experimental requirement, so that the total measurement times $P_{L M}$ and $P_{C W}$ have a ratio of $\sqrt{ } 2$.

Eq. A.1 makes the time axis of both LM and ps-LM OSL to have the same values. However, in order for the LM-OSL and the ps-LM-OSL curves to coincide exactly, their peak time maxima must also coincide. The time maximum for a first order LM-OSL peak is given by the equation (Bulur 1996)

$t_{m}^{2}=\frac{P_{L M}}{\alpha I_{L M}}$

and with a similar expression for the corresponding psLM-OSL peak shaped data:

$$
u_{m}^{2}=\frac{P_{P S-L M}}{\alpha I_{P S-L M}}
$$

Therefore by setting $u_{m}=t_{m}$ one has from Eqs. A.2 and A.3:

$$
\frac{P_{P S-L M}}{I_{P S-L M}}=\frac{P_{L M}}{I_{L M}} .
$$

Finally by taking into account Eq. A.1 we obtain the necessary condition for the LM-OSL maximum to occur at the same time value as the peak-shaped ps-LM-OSL data:

$I_{L M}=\sqrt{2} \cdot I_{C W}$.

Under the settings given by Eqs. A.1 and A.5, the LM-OSL and ps-LM-OSL curves should coincide exactly in shape. However, the integrals under the peaks will be different due to the transformation applied to the CWOSL data.

In the present work we chose the total stimulation times to be $P_{C W}=355 \mathrm{~s}$ for the CW-OSL measurements and $P_{L M}=\sqrt{2} P_{C W}=\sqrt{ } 2(355 \mathrm{~s})=500 \mathrm{~s}$ for the LM-OSL measurements. Similarly, the LED powers were chosen to have a ratio of $\sqrt{2}$, namely 28 and $40 \mathrm{~mW} / \mathrm{cm}^{2}$ for the $\mathrm{CW}-\mathrm{OSL}$ and LM-OSL measurements respectively.

A comparison between the LM-OSL and CW-OSL signals also requires that the total light energy delivered to the sample be equal for the two modes of optical stimulation. The total CW-OSL and LM-OSL energy delivered to the sample are given by Eqs. 3.2b and 3.5b correspondingly. By using the settings given by Eqs. A.1 and $\mathbf{A . 5}$, it is easy to show that the two total energies are indeed the same:

$$
E_{C W}=I_{C W} P_{C W}=\frac{I_{L M}}{\sqrt{2}} \frac{P_{L M}}{\sqrt{2}}=\frac{1}{2} I_{L M} P_{L M}=E_{L M} \text { (A.6) }
$$

